\title{
Irritant contact facial dermatitis due to nebulizer therapy
}

\author{
David J. Eedy, Keith Barton, ${ }^{1}$ and C.F. Stanford ${ }^{1}$ \\ Department of Dermatology and ${ }^{1}$ Respiratory Unit, Royal Victoria Hospital, Belfast BT12 6BJ, UK.
}

\begin{abstract}
Summary: A case is reported of a patient with long-standing, severe airflow obstruction requiring long-term nebulizer therapy developing a facial dermatitis in the area bounded by the nebulizer mask. The facial dermatitis seems to be the result of the combined irritancy of the nebulizer solutions and moisture, and prophylactic measures are suggested for patients requiring long-term nebulizer therapy.
\end{abstract}

\section{Introduction}

Despite the widespread use of nebulizers in the treatment of obstructive airways disease, there appear to be few observed side effects in relation to the facial skin. Pharyngeal blistering has recently been reported with the combined use of ipratropium bromide (Atrovent) and terbutaline sulphate (Bricanyl) inhalers ${ }^{1}$ and buccal ulceration has been reported with inhaled ipratropium bromide. $^{2}$ We report a case of irritant contact dermatitis with superimposed staphylococcal impetigo occurring on the facial skin of a patient receiving regular nebulizer therapy with ipratropium bromide and terbutaline sulphate.

\section{Case report}

A 53 year old man with severe airflow obstruction of several years duration was admitted to hospital for intensive nebulizer therapy. He developed a facial dermatitis with superimposed staphylococcal impetigo 6 weeks after commencement of nebulizer therapy with the combination of ipratropium bromide (Atrovent) and terbutaline sulphate (Bricanyl). The eczematous reaction involved the central face in a 'muzzle distribution' in the area bounded by the nebulizer mask and there were sharp cut-off margins suggesting a contact dermatitis (Figure 1). This photograph was taken after several days of treatment when significant clinical improvement had occurred. There was no history of previous skin problems and on examination, no rashes noted elsewhere. Bacteriology swabs taken from the affected area and nose cultured Staphylococcus aureus but

Correspondence: D.J. Eedy, M.B., B.Ch., M.R.C.P. Accepted: 23 November 1987 staphylococci were not cultured from the humidity bottle of the nebulizer. The intranasal carrier sites were cleared using topical soframycin cream and the facial rash was treated initially with povidoneiodine (Betadine) paint and polymyxin B and bacitracin (Polyfax) ointment four times daily for 5 days before changing to $1 \%$ hydrocortisone and miconazole 2\% cream (Daktacort) three times daily. The facial rash cleared within a further 10 days after which the facial skin was protected with soft white paraffin while the nebulizer therapy was allowed to continue. There has been no recurrence of the facial rash using this regime after 12 weeks.

Patch tests were applied to the standard European battery, plastics and glues (Chemotechnique Diagnostics Batteries), scrapings from the plastic of the nebulizer mask and both $5 \%$ and $10 \%$ solutions of ipratropium bromide (Atrovent) and terbutaline sulphate (Bricanyl), and the patch test read at 72 hours. All the patch tests were negative.

\section{Discussion}

This case describes the occurrence of an irritant contact dermatitis occurring in a patient requiring long-term use of nebulized ipratropium bromide and terbutaline sulphate. The facial dermatitis occurred within the area bounded by the nebulizer mask and we believe this to be due to the combined irritancy effects of the nebulized bronchodilator solutions and moisture on the skin. It is surprising that this is not a commonly encountered problem in view of the widespread use of nebulized bronchodilators and we are unaware of similar reports. It is interesting that irritancy has been associated with the inhaler formulations of these drugs. ${ }^{1,2}$ Allergic contact dermatitis has been associated with rubber

(C) The Fellowship of Postgraduate Medicine 1988 


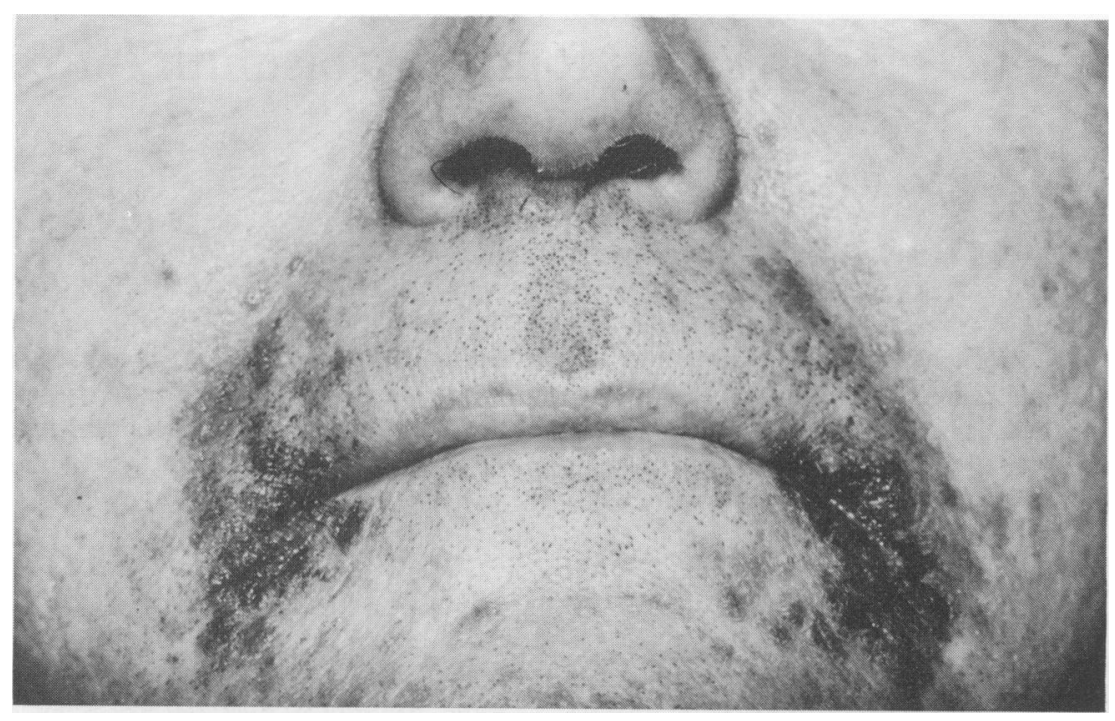

Figure 1 Distribution of facial rash several days after commencing treatment.

face masks ${ }^{3}$ where patch testing confirmed the diagnosis and where the pattern of dermatitis on the face traces out the areas in contact with the mask. ${ }^{4}$ In this case allergy to the face mask and the nebulizer drugs was not a feature.

The prophylactic use of a preparation such as soft white vaseline, as well as the eradication of

\section{References}

1. High, A.S. Pharyngeal blistering with combined inhaler therapy. $B r$ Med J 1987, 294: 375.

2. Spencer, P.A. Buccal ulceration with ipratropium bromide. Br Med J 1986, 292: 380.

3. Maibach, H.I. Scuba diver facial dermatitis: allergic nasal pathogens with an appropriate intranasal antibiotic, may be worth considering in patients who require frequent and protracted periods of nebulizer therapy. With the increasing use of home nebulizers if would also seem prudent to advise patients to keep face masks as clean as possible to reduce the risks of secondary bacterial infection.

contact dermatitis to $N$-isopropyl- $N$-phenylparaphenylenediamine. Contact Dermatitis 1975, 1: 330

4. Cronin, E. Rubber. In: Contact Dermatitis. Churchill Livingstone, Edinburgh, 1980, pp 761-762. 